\title{
Acute pancreatitis complicated by rupture of abdominal aortic aneurysm
}

\author{
Krzysztof Wachal ${ }^{1}$, Zbigniew Krasiński ${ }^{1}$, Krzysztof Szmytt ${ }^{1}$, Jacek Białecki ${ }^{2}$, Sylwia Sławek ${ }^{2}$, Grzegorz Oszkinis ${ }^{1}$ \\ ${ }^{1}$ Department and Clinic of General and Vascular Surgery, Poznan University of Medical Sciences, Poznan, Poland \\ 2Department of General and Miniinvasive Surgery, Franciszka Raszei Memorial Hospital, Poznan, Poland
}

Gastroenterology Rev 2016; 11 (2): 136-138

DOI: $10.5114 / p g .2016 .57820$

\begin{abstract}
Address for correspondence: Krzysztof Wachal MD, PhD, Department and Clinic of General and Vascular Surgery, Poznan University of Medical Sciences, 1/2 Długa St, 61-848 Poznan, Poland, phone: +48 604570 717, e-mail: krzywach@poczta.fm
\end{abstract}

Acute pancreatitis (AP) is a potentially life-threatening inflammatory disease with wide ranging severity and unpredictable evolution [1]. Extremely rarely AP co-exists with abdominal aortic aneurysm (AAA). It is estimated that, among gastroenterological complications associated with ruptured abdominal aortic aneurysm ( $\mathrm{rAAA}$ ), AP occurs in $0.7 \%$ and significantly increases the mortality rate [2]. However, rupture of AAA caused by elevated pancreatic enzymes concentrations has not been previously described. This case is the first documenting rAAA in the course of severe AP. We present a case of severe AP complicated by rupture of AAA, which was treated by open abdomen (OA) with application of negative pressure wound therapy (NPWT).

A 65-year-old patient was admitted to the surgical ward because of severe pain in the upper abdomen with nausea and vomiting without relief for $10 \mathrm{~h}$. Laboratory tests revealed the following: elevated serum amylase, serum lipase, C-reactive protein (CRP), white blood cell count, and serum glucose. Ultrasound examination demonstrated the presence of AAA localised below the renal arteries, measuring $7 \times 5 \mathrm{~cm}$, without evidence of rupture. A diagnosis of AP was made. On admission, the severity of AP on the Ranson scale was assessed as 3. Conservative therapy was initiated. During the fourth day of hospitalisation, the patient's condition deteriorated, epigastric pain had intensified, and clinical symptoms of the hypovolemic shock occurred. Computed tomography (CT) confirmed raptured rAAA.

According to APACHE II scale the severity of AP was classified on 14 scores (severe AP). Computed tomography angiography (angio-CT) indicated rAAA with dimensions $8 \times 5.8 \mathrm{~cm}$ (Figure 1). Pancreatic parenchyma was assessed according to CTSI index on 2 points. None of acute peripancreatic fluid collections (APFC) were identified. Emergency surgery of the rAAA was performed.

During the operation a linear $1.5-\mathrm{cm}$ rupture of the infrarenal AAA sac was indicated. In place of the rAAA a simple aortic prosthesis was sutured (length of prosthesis $80 \mathrm{~mm}$, Cook). About $1800 \mathrm{ml}$ of blood was sucked from the abdominal cavity. Immediately after surgery an ABThera dressing ( $\mathrm{KCl}$ company, San Antonio, USA) was applied (Figures 2, 3).

The duration of NPWT was $30 \mathrm{~h}$, and the dressing was changed two times (respectively, after 10 and $20 \mathrm{~h}$ ). A negative pressure of $125 \mathrm{~mm} \mathrm{Hg}$ was applied in continuous mode. Up to the time of the first dressing change $350 \mathrm{ml}$ of fluid was resorbed from the abdominal cavity, and up to the second dressing exchange it was $120 \mathrm{ml}$. In the immediate postoperative period, intra-abdominal pressure (IAP) was measured in the bladder, to prevent the development of intra-abdominal hypertension (ACS). The highest score of IAP was $15 \mathrm{~mm} \mathrm{Hg}$. Renal, heart, and liver function markers were controlled. Angio-CT performed on the $7^{\text {th }}$ day after surgery confirmed the correct implantation of the prosthesis without the presence of leakage into the aneurysm sac and normal pancreas image. Contrast-enhanced ultrasonography (CEUS) investigation made on the $13^{\text {th }}$ day after hospitalisation did not show abnormalities.

The patient underwent primary surgical wound suturing. Good results of AP treatment were achieved and there was no presence of ACS or secondary haemorrhage, or infection. The patient was discharged to his home in good general condition on the $14^{\text {th }}$ day. During the 6-month postoperative follow-up at the Department of General and Vascular Surgery no clinical and radiological symptoms of prosthesis infection has occurred. 
Histologically, AAA is characterised by vessel adventitia inflammation, tunica media thinning, and loss of elastic fibres [3]. The aetiology of AP as rAAA complication is unclear. James et al. proposed the theory of post-operative microemboli formation in pancreatic vascularity, which leads to pancreatic ischaemia [4]. The most important risk factors for the development of AP after AAA repair surgery include an urgent operation mode, time of the aorta clamping and organs ischaemia, and intraoperative mechanical pancreas damage [5]. An important role is alos played by the co-occurrence of diabetes mellitus [6]. It is noted that an increase of serum amylase concentration usually occurs in patients with mild AP, while in patients with severe AP amylase concentration is usually normal. However, in patients with AAA serum amylase and lipase concentrations may be significantly increased, irrespective of the functional pancreas state, and this increases with the size of the aneurysm (two times more frequent when the diameter of aneurysm sac is $>7 \mathrm{~cm}$ ) [7].

The dilatation of arterial lumen in the form of pseudoaneurysms of splenic, pancreatoduodenal, or gastroduodenal arteries is a relatively frequent AP complication. Pseudoaneurysm occurs in $7-31 \%$ of cases [8]. Aortic aneurysm formation has not been described.

Lysis is the main mechanism of blood vessel damage. Prematurely activated pancreatic enzymes, mainly elastase digest elastic fibres of tunica media, lead to a disruption of the vessel wall or a significant weakening of its structure, dissection, and aneurysm formation. A series of reports in animal models support the role of enzymes in the pathogenesis of pseudoaneurysms and aneurysms. Bi et al. induced AAA after the administration of elastase to infrarenal aorta of $1.5 \mathrm{~cm}$ length, on the fifth day of the experiment [3]. Inflammatory response following administration of elastase is accompanied by increased production of macrophage metalloproteinases. Pyo et al. demonstrated a significant role of metalloproteinase 9 (MMP-9, gelatinase B) and metalloproteinase 12 (MMP-12, macrophage elastase) in the development of AAA [9]. Macrophage elastase is thought to play a much more important role in the development of AAA than pancreatic elastase [9]. This is due to the fact that pancreatic enzymes are not present in significant quantities in the circulation outside episodes of AP. It remains unexplained why aortic wall injuries in the course of AP are rare. Hinchliffe et al. suggested the presence of protective factors against proteolytic enzymes activity in aortic adventitia [10]. In the described case, the rupture of AAA appeared in the course of AP, which proves that the wall of the aorta weakened by the presence of an aneurysm is sensitive to proteolytic enzymes released from the inflamed pancreas and activated macrophages.

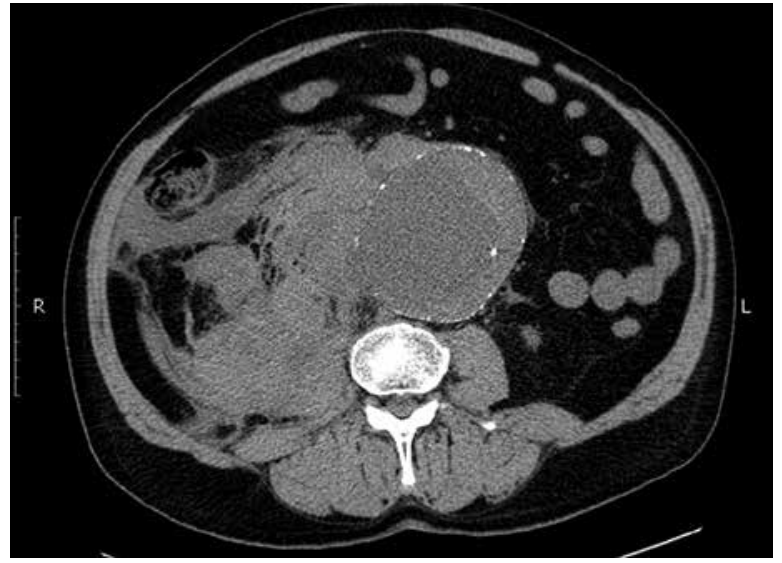

Figure 1. The preoperative computed tomography - maximum diameter of the aneurysm sac was about $8 \mathrm{~cm}$

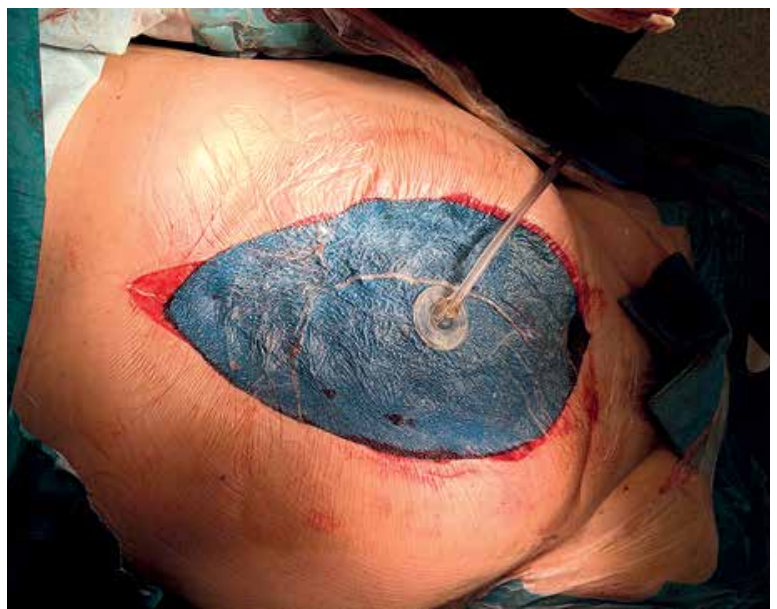

Figure 2. Status after surgical procedure and application of ABThera dressing

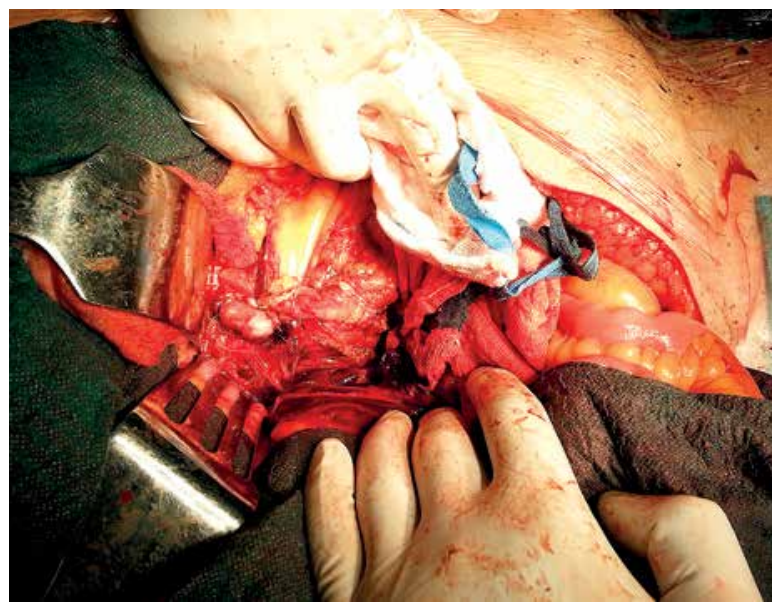

Figure 3. Status after removing NPWT dressing (24 h) 
It is estimated that ACS after rAAA surgery occurs in $19 \%$ of cases, with $66 \%$ mortality [11]. Currently, the most effective technique in ACS prevention is OA. Application of OA allows the avoidance of decompression laparotomy in patients with IAH. Negative pressure wound therapy has additional antibacterial properties and stimulates the formation of granulation tissue. Moreover, the use of NPWT is associated with a lower risk of fistula or abdominal hernia in postoperative scar.

\section{Conflict of interest}

The authors declare no conflict of interest.

\section{References}

1. Koziel D, Kozlowska M, Deneka J, et al. Retrospective analysis of clinical problems concerning acute pancreatitis in one treatment center. Prz Gastroenterol 2013; 8: 320-6.

2. Drissi M, Madani M, Hatim A, et al. Severe acute pancreatitis after surgical treatment of a ruptured abdominal aortic aneurysm. Ann Vasc Surg 2009; 23: 785 e785-7.

3. Bi Y, Zhong H, Xu K, et al. Development of a novel rabbit model of abdominal aortic aneurysm via a combination of periaortic calcium chloride and elastase incubation. PLoS One 2013; 8: e68476.

4. James AD, Anderson HJ, Edwards R, et al. Pancreatitis as a complication of endovascular aneurysm repair. Eur J Vasc Endovasc Surg 2008; 35: 310-1.

5. Hashimoto L, Walsh RM. Acute pancreatitis after aortic surgery. Am Surg 1999; 65: 423-6.

6. Rayan SS, Hamdan AD, Campbell DR, et al. Is diabetes a risk factor for patients undergoing open abdominal aortic aneurysm repair? Vasc Endovascular Surg 2002; 36: 33-40.

7. Krasiński Z, Dziedziuchowicz Ł, Stanisic M, et al. Pancreatic injury following elective abdominal aortic repair. Acta Angiologica 2005; 11: 204-11.

8. Marshall GT, Howell DA, Hansen BL, et al. Multidisciplinary approach to pseudoaneurysms complicating pancreatic pseudocysts. Impact of pretreatment diagnosis. Arch Surg 1996; 131: 278-83.

9. Pyo R, Lee JK, Shipley JM, et al. Targeted gene disruption of matrix metalloproteinase-9 (gelatinase B) suppresses development of experimental abdominal aortic aneurysms. J Clin Invest 2000; 105: 1641-9.

10. Hinchliffe RJ, Yung M, Hopkinson BR. Endovascular exclusion of a ruptured pseudoaneurysm of the infrarenal abdominal aorta secondary to pancreatitis. J Endovasc Ther 2002; 9: 590-2.

11. Starnes BW, Quiroga E, Hutter C, et al. Management of ruptured abdominal aortic aneurysm in the endovascular era. J Vasc Surg 2010; 51: 9-17; discussion 17-8.

Received: 12.02 .2015

Accepted: 14.06.2015 